\title{
Physical and functional interaction between viral and cellular proteins modulate JCV gene transcription
}

\author{
Mahmut Safak and Kamel Khalili \\ Center for Neurovirology and Cancer Biology, Temple University, Philadelphia, Pennsylvania, USA
}

\begin{abstract}
The lytic phase of JC virus (JCV) appears to be highly complex and remains elusive. A growing body of experimental evidence suggests that the regulation of JCV gene expression and replication requires, in addition to the presence of specific transcription factors, cooperativity between viral and cellular regulatory proteins. This cooperativity may be accomplished by physical interaction of the participant proteins on and/or off the viral DNA sequence. Here, we present evidence of specific physical and functional interaction between a cellular factor, YB-1, and the JCV early protein, T-antigen, and showed that both proteins play important roles in JCV gene transcription. Additionally, our data indicate that YB-1 also functionally interact with another viral protein, designated agnoprotein, which is expressed late during the course of infection, adding further complexity to the currently known picture on JCV gene regulation. Journal of NeuroVirology (2001) 7, 288-292.
\end{abstract}

Keywords: agnoprotein; interaction; T-antigen; YB-1

Human polyoma virus, JC virus (JCV), is a small DNA virus with a double-stranded covalently linked circular genome. It is the causative agent of progressive multifocal leukoencephalopathy where oligodendrocytes, the myelin-producing cells of central nervous system, are selectively destroyed by this virus. JCV genome is composed of three functional regions including the viral early-, late-coding regions, and noncoding regulatory region (Frisque et al, 1984). The viral early- and late-coding regions encode regulatory (large and small t-antigens) and structural (VP1, -2, and -3) proteins, respectively. The viral late genome also contains an open-reading frame for a small auxiliary protein called agnoprotein, whose function in the viral life cycle is virtually unknown. The viral noncoding regulatory region contains the origin of DNA replication, promoter elements for both early and late genes, and cis-acting enhancer elements, which are characterized by two 98-bp tandem repeats.

Transcriptional regulation of the JCV early and late promoters in the lytic cycle appears to be rather

Address correspondence to Dr. Kamel Khalili, Center for Neurovirology and Cancer Biology, College of Science and Technology, Temple University, 1900 North 12th Street, 015-96, Room 203, Philadelphia, PA 19122, USA. E-mail: kkhalili@astro.temple.edu

Received 7 March 2001; revised 22 March 2001; accepted 10 April 2001. complex and requires both participation of viral and cellular factors. The JCV lytic cycle begins with the expression of early genes encoding the regulatory large and small t-antigens. Large T-antigen, the central regulator of the viral lytic cycle, initiates viral DNA replication and orchestrates the transition from early to late gene transcription by activating late gene expression and by suppressing its own early promoter (Khalili et al, 1987; Lashgari et al, 1989). Following the onset of DNA replication, the virus enters the late phase of infection during which capsid proteins are encoded, virions are matured, and finally the host cell is lysed (Raj and Khalili, 1995). Recently, we have presented evidence that, like large T-antigen, agnoprotein of JCV also participates in regulation of viral DNA replication and transcription (Safak et al, 2001).

Over the years, a number of cellular transcription factors have been identified and characterized for their involvement in JCV gene transcription including NF- $\kappa$ B (Ranganathan and Khalili, 1993; Safak et al, 1999b), Tst-1 (Wegner et al, 1993; Renner et al, 1994), NF-1 (Amemiya et al, 1989), Sp-1 (Henson et al, 1992), GBP-i (Raj and Khalili, 1994), YB-1 (Kerr et al, 1994; Safak et al, 1999a), and Pur $\alpha$ (Chen and Khalili, 1995; Chen et al, 1995; Safak et al, 1999c). At the initial stages of infection, only host cellular transcription factors are responsible for 
expression of early genes in the absence of T-antigen. At later stages of infection, particularly molecular interactions between host and viral proteins appears to take the central stage in governing both viral DNA replication and transcription. Among the previous cellular transcription factors, we have particularly focused our attention to the molecular mechanism(s) of the transcriptional regulation of JCV promoters by cellular factor, YB-1, through its interaction with viral early regulatory protein, large T-antigen, and with late regulatory protein, agnoprotein. YB-1 is a member of the large family of Y-box DNA binding proteins, whose (Wolffe, 1994) family members are conserved from bacteria to higher eukaryotes. All vertebrate Y-box binding proteins consist of three domains: a variable glycine-rich $\mathrm{N}$-terminus, a highly conserved central nucleic acid recognition domain, and a hydrophilic C-terminus tail domain. Central nucleic acid recognition domain is also known as the cold-shock domain and shows $43 \%$ homology to the bacterial cold-shock protein (Lee et al, 1994). The tail domain, which is thought to stabilize protein-DNA interactions, contains alternating regions of predominantly basic or acidic amino acids. Compelling experimental evidence indicates that Y-box binding proteins are involved in a wide variety of biological functions including regulation of gene expression at both transcriptional (Wolffe, 1994; Mertens et al, 1997; Safak et al, 1999a, 1999c) and translational levels (Tafuri and Wolffe, 1993); DNA and RNA condensation, DNA repair, and stress-induction (Bargou et al, 1997; Koike et al, 1997).

To investigate the effect of YB-1 on basal and T-antigen-induced transactivation of the JCV promoters, we performed transient transfection experiments in the human glial cell line, U-87MG, using reporter constructs containing the JCV early and late gene promoters. As shown in Figure 1A, cotransfection of the reporter construct with a T-antigen expression plasmid resulted in a substantial increase in the transcriptional activity from the late promoter (8- to 9-fold increase, compare lanes 1 and 2). However, when a constant amount of T-antigen was cotransfected with an increasing amount of YB-1, we observed a synergistic effect on the transcriptional activity of the late promoter (14- to 18-fold increase, compare lane 2 to 3 and 4). At the highest YB-1 concentration alone, we observed only a 3 -fold increase in transcription (Figure 1A, lane 5), demonstrating a transcriptional synergy between these two transactivators on transcription from the late promoter. Of note, expression of T-antigen or YB-1 remains unaltered upon expression of either transactivator (data not shown), suggesting that transcriptional cooperativity observed between T-antigen and YB-1 is not due to the effect of one transactivator on the expression of the other.

Similarly, we also carried out cotransfection assays to evaluate the transcriptional cooperation between
YB-1 and T-antigen on the viral early promoter. As shown in Figure 1B, a CAT reporter construct containing the JCV early promoter showed a notable basal expression in the absence of transactivators (lane 1). However, as expected, cotransfection of the reporter construct with the T-antigen expression plasmid resulted in a substantial decrease in transcriptional activity, demonstrating the T-antigenmediated transcriptional suppression of the early promoter (compare lanes 1 and 2). In contrast, when a constant amount of T-antigen was cotransfected with an increasing amount of YB-1, we observed a dose-dependent alleviation in the T-antigenmediated transcriptional suppression of the early promoter (compare lane 2 to 3 and 4), again indicating the presence of a functional interaction between YB-1 and T-antigen. As expected, YB-1 alone significantly activates transcription from early promoter (lane 5).

In addition to functional interaction observed between YB-1 and T antigen, we also investigated direct physical interaction between these two proteins utilizing in vitro GST (glutathione- $S$-transferase)pulldown assays and localized the interaction domain of YB-1 with large T-antigen (Safak et al, 1999b). A series of amino- and carboxy-terminal deletion mutants of YB-1 was created as GST fusion proteins and incubated with whole cell extracts prepared from hamster glial cells (HJC) constitutively expressing JCV large T-antigen. Unbound proteins were washed extensively with binding buffer and bound proteins were analyzed by Western blot analysis with antibodies specific for T-antigen. As shown in Figure 1C, results from these assays showed that YB-1 and T-antigen physically interacts, and T-antigen interaction domain of YB-1 is localized to its cold shock domain and immediate surrounding residues.

We have recently presented evidence that JCV agnoprotein can physically and functionally interact with the viral early protein, T-antigen and downregulate both T-antigen-mediated viral gene expression and DNA replication (Safak et al, 2001). Because cellular factor YB-1 participates in JCV gene regulation through its interaction with both cellular factors (Chen et al, 1995; Safak et al, 1999c) and JCV T-antigen (Safak et al, 1999a), we sought to investigate whether this factor is also involved in JCV gene regulation through its interaction with viral late regulatory protein, agnoprotein. We performed cotransfection experiments in U-87MG cells, using a reporter construct containing the JCV early gene promoter and expression plasmids for YB-1 and agnoprotein. As shown in Figure 2A, YB-1-induced expression of viral early promoter is substantially suppressed by agnoprotein in a dose-dependent manner (lanes 3 and 4), indicating the functional interaction between these two proteins. As previously observed for viral late promoter (Safak et al, 2001), agnoprotein also suppressed the basal expression of viral early 

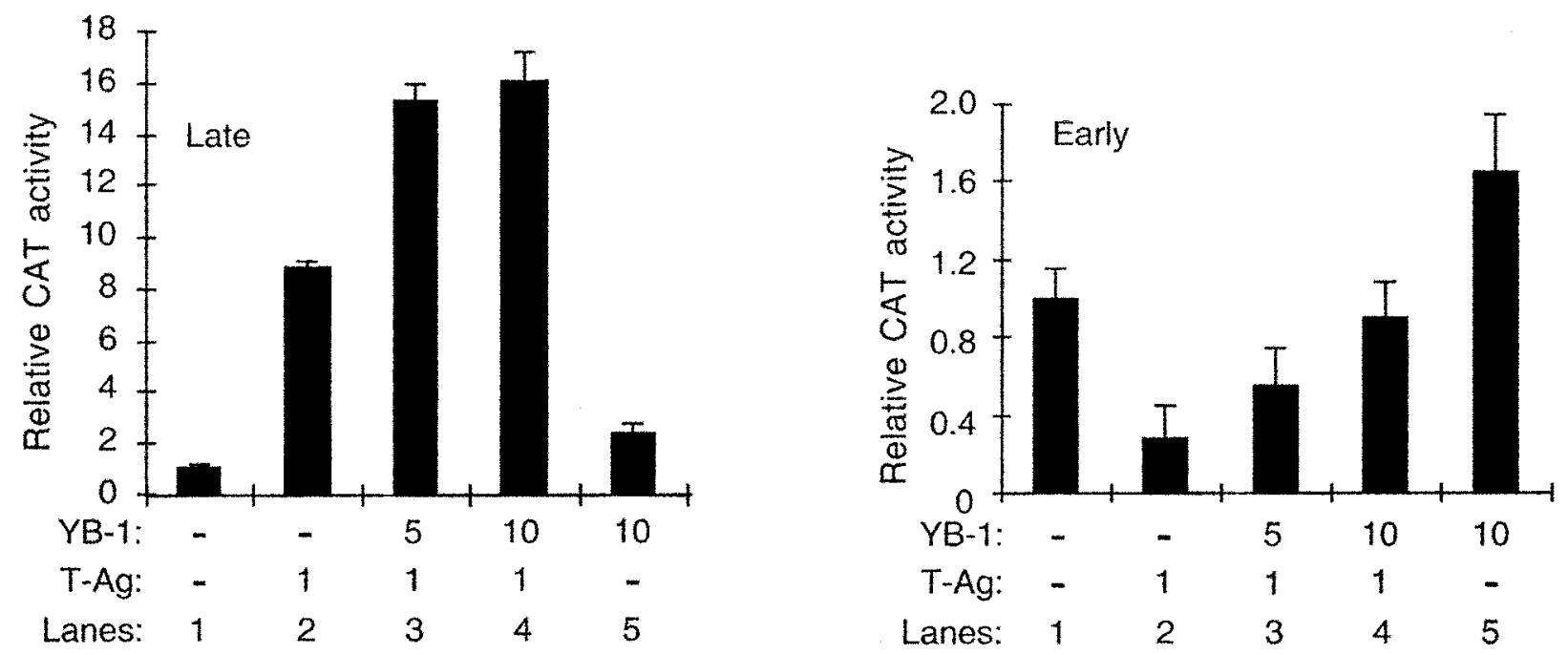

C

Glycine-rich

DNA binding

Acidic and basic repeats

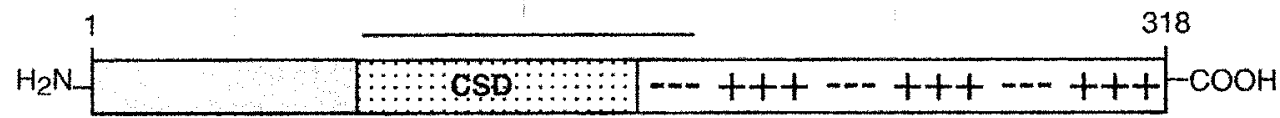

T-Ag binding

YB-1 deletion mutants

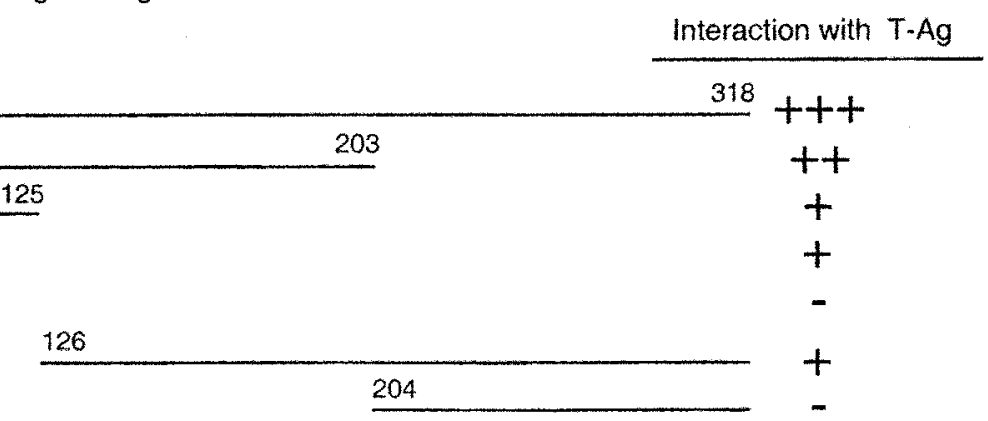

Figure 1 Functional and physical interaction between YB-1 and T-antigen. (A) A reporter plasmid (pBLCAT $\left.{ }_{3}-\mathrm{Mad}_{-} \mathrm{L}\right)(7.5 \mu \mathrm{g}) \mathrm{containing}$ the JCV late gene promoter (late) was transfected into U-87MG cells alone or together with EBV-YB-1 and CMV-T antigen expression plasmids. Expression plasmid concentrations were normalized with appropriate empty vectors. At 36 h posttransfection, cells were harvested and cloramphenicol acetyl transferase (CAT) activity of extracts were determined using equal amount of protein from each transfectant. CAT activities were presented as CAT activity relative to the basal activity of the promoter. (B) Experiments similar to those

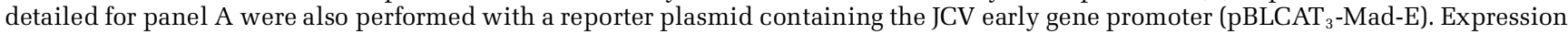
plasmid DNA concentrations used in each transfection are indicated at the bottom of the each panel in micrograms. Bars indicate standard deviation. (C) A summary of results obtained from GST-pulldown assays. A schematic representation of full-length YB-1 is shown at the top. The ability of YB-1 and its deletion mutants to interact with T-antigen is depicted on the right as +++ , specific interaction; ++ or + , reduced interaction; and - , no interaction.

promoter (lane 5). Additionally, our preliminary cotransfection experiments indicate that agnoprotein also appears to suppress YB-1-mediated transactivation from JCV late promoter (data not shown). These observations suggested that YB-1 may physically interact with JCV agnoprotein. To test this possibility, we performed affinity chromatography (GST pulldown) experiments. GST or GST-Agno protein was immobilized on glutathione-sepharose beads and incubated with whole cell extracts from HJC-15b cells, transfected with histidine-tagged YB-1. Proteins bound to beads were extensively washed and analyzed by Western blot analysis using antibodies specific for histidine-tagged YB-1. As demonstrated in Figure 2B, YB-1 was specifically retained by the sepharose column containing GST-Agno (compare lane 4 with 3).

In conclusion, we have presented evidence for physical and functional interactions between a cellular factor, YB-1, and two viral proteins, viral early 

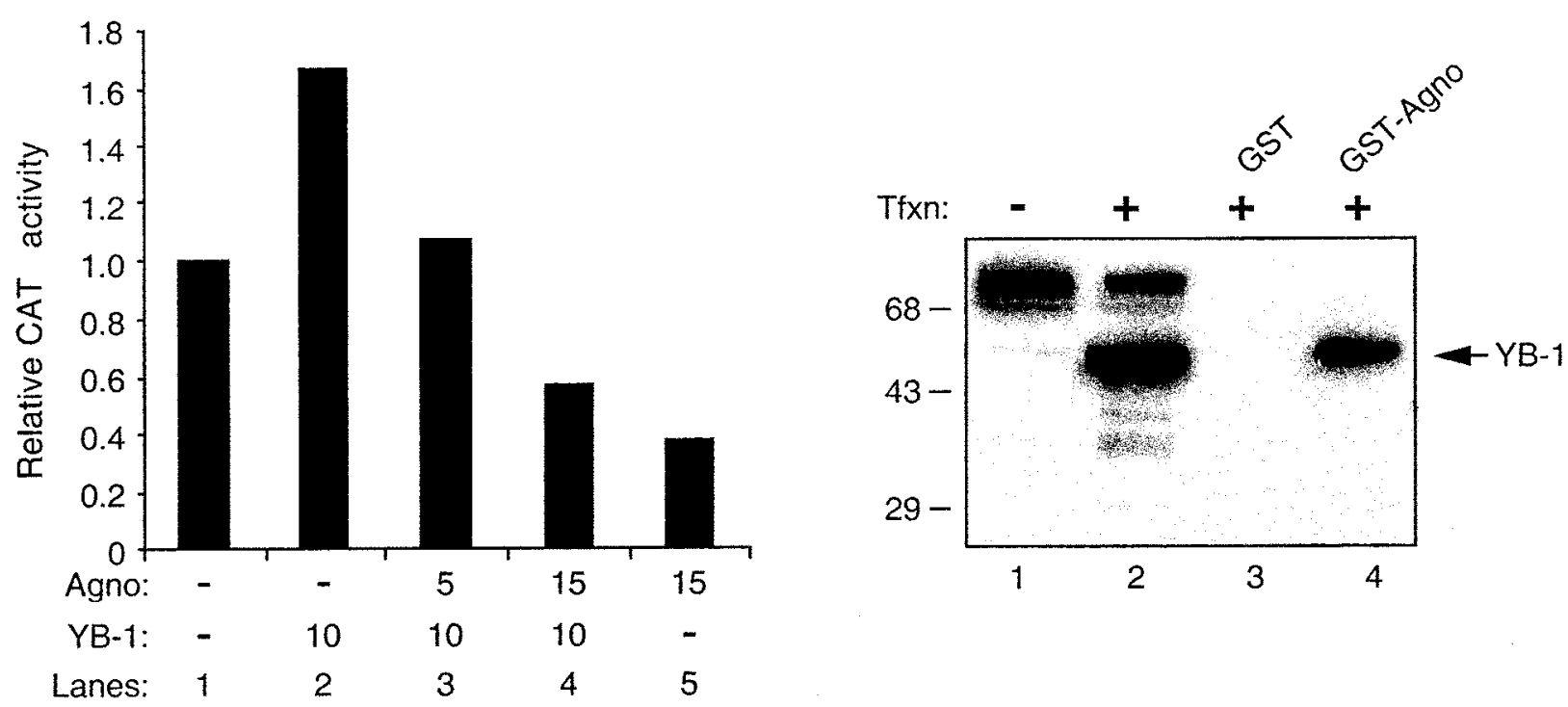

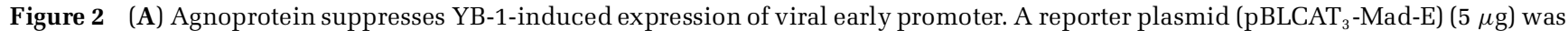
transfected into U-87MG cells alone or together with CMV-Agno and EBV-YB-1 expression plasmids. CAT activities for each transfectant was determined as detailed for Figure 1A. (B) In vitro interaction of YB-1 with agnoprotein. Whole-cell extracts prepared from HJC-15b cells transfected with a histidine-tagged YB-1 expression plasmid (pEBV-YB-1) (lanes 3 and 4) were incubated with either GST alone (lane 3) or GST-Agno (lane 4) as indicated. Beads were washed extensively and proteins interacting with GST or GST-Agno were analyzed by Western blot analysis using anti-T7 antibody for detection of His-tagged YB-1. Whole cell extract from HJC-15b cells either untransfected (lane 1) or transfected with pEBV-His-YB-1 expression plasmid (lane 2) were loaded as negative and positive migration controls respectively. Agno designates agnoprotein. Tfxn indicates transfection.

regulatory protein, large T-antigen, and viral late regulatory agnoprotein. Characterization of such delicate interactions at the molecular level will further our understanding of molecular mechanism(s)

\section{References}

Amemiya K, Traub R, Durham L, Major EO (1989). Interaction of a nuclear factor-1-like protein with the regulatory region of the human polyomavirus JC virus. J Biol Chem 264: 7025-7032.

Bargou RC, Jurchott K, Wagener C, Bergmann S, Metzner S, Bommert K, Mapara MY, Winzer KJ, Dietel M, Dorken B, Royer HD (1997). Nuclear localization and increased levels of transcription factor YB-1 in primary human breast cancers are associated with intrinsic MDR1 gene expression [see comments]. Nat Med 3: 447450.

Chen NN, Chang C-F, Gallia GL, Kerr DA, Johnson EM, Krachmarov CP, Barr SM, Frisque RJ, Bollag B, Khalili $\mathrm{K}$ (1995). Cooperative action of cellular proteins YB-1 and Pur alpha with the tumor antigen of the human JC polyomavirus determines their interaction with the viral lytic control element. Proc Natl Acad Sci USA 92: 1087-1091.

Chen NN, Khalili K (1995). Transcriptional regulation of human JC polyomavirus promoters by cellular proteins YB-1 and Pur alpha in glial cells. J Virol 69: 5843-5848.

Didier DK, Schiffenbauer J, Wolffe SL, Zacheis M, Schwartz $\mathrm{BD}$ (1988). Characterization of the cDNA encoding a protein binding to the major histocompatibility complex class II Y box. Proc Natl Acad Sci USA 85: 7322-7326. governing the JC virus lytic cycle and may eventually help us to design effective molecular therapeutics against JC virus infections before the virus enters its lytic cycle.
Frisque RJ, Bream GL, Cannella MT (1984). Human polyomavirus JC virus genome. J Virol 51: 458-469.

Henson J, Saffer J, Furneaux H (1992). The transcription factor Sp1 binds to the JC virus promoter and is selectively expressed in glial cells in human brain. Ann Neurol 32: 72-77.

Kerr D, Chang C-F, Chen N, Gallia GL, Raj GV, Schwartz B, Khalili K (1994). Transcription of a human neurotropic virus promoter in glial cells: effect of YB-1 on expression of the JC virus late gene. J Virol 68: 76377643.

Khalili K, Feigenbaum L, Khoury G (1987). Evidence for a shift in $5^{\prime}$-termini of early viral RNA during the lytic cycle of JC virus. Virology 158: 469-472.

Koike K, Uchiumi T, Ohga T, Toh S, Wada M, Kohno K, Kuwano M (1997). Nuclear translocation of the Y-box binding protein by ultraviolet irradiation. FEBS Lett 417: 390-394.

Lashgari M, Tada H, Amini S, Khalili K (1989). Regulation of JCVL promoter function. Transactivation of JCVL promoter by JCV and SV40 early proteins. Virology 170: 292-295.

Lee SJ, Xie A, Jiang IM, Etchegaray JP, Jones PG, Inouye M (1994). Family of the major cold-shock protein, CspA (CS 7.4), of Escherichia coli, whose members show 
a high sequence similarity with the eukaryotic Y-box binding proteins. Mol Microbiol 5: 833-839.

Mertens PR, Harendza S, Pollock AS, Lovett DH (1997). Glomerular mesangial cell-specific transactivation of matrix metalloproteinase 2 transcription is mediated by YB-1. J Biol Chem 272: 22905-22912.

Raj GV, Khalili K (1994). Identification and characterization of a novel GGA/C-binding protein, GBP-i, that is rapidly inducible by cytokines. Mol Cell Biol 14: 7770-7781.

Raj GV, Khalili K (1995). Transcriptional regulation: lessons from the human neurotropic polyomavirus, JCV. Virology 213: 283-291.

Ranganathan PN, Khalili K (1993). The transcriptional enhancer element, kappa B, regulates promoter activity of the human neurotropic virus, JCV, in cells derived from the CNS. Nucleic Acids Res 21: 1959-1964.

Renner K, Leger H, Wegner M (1994). The POU doamin protein Tst-1 and papovaviral large tumor antigen function synergistically to stimulate glia-specific gene expression of JC virus. Proc Natl Acad Sci USA 91: 6433-6437.

Safak M, Barrucco R, Darbinyan A, Okada Y, Nagashima K, Khalili K (2001). Interaction of JC virus Agno protein with $\mathrm{T}$ antigen modulates transcription and replication of the viral genome in glial cells. J Virol 75: 1476-1486.
Safak M, Gallia GL, Ansari SA, Khalili K (1999a). Physical and functional interaction between the Y-box binding protein YB-1 and human polyomavirus JC virus large T antigen. J Virol 73: 10146-10157.

Safak M, Gallia GL, Khalili K (1999b). A 23-bp sequence element from human neurotropic JC virus is responsive to NF-kappa B subunits. Virology 262: 178189.

Safak M, Gallia GL, Khalili K (1999c). Reciprocal interaction between two cellular proteins, Puralpha and YB1 , modulates transcriptional activity of JCVCY in glial cells. Mol Cell Biol 19: 2712-2723.

Tafuri SR, Wolffe AP (1993). Selective recruitment of masked maternal mRNA from messenger ribonucleoprotein particles containing FRGY2 (mRNP4). J Biol Chem 268: 24255-24261.

Wegner M, Drolet DW, Rosenfeld MG (1993). Regulation of JC virus by the POU-domain transcription factor Tst-1: Implications for progressive multifocal leukoencephalopathy. Proc Natl Acad Sci USA 90: 97439747

Wolffe AP (1994). Structural and functional properties of the evolutionarily ancient Y-box family of nucleic acid binding proteins. Bioessays 16: 245-251. 